\title{
Diagnostic Value of Lung Ultrasound Versus Chest X-Ray in Surgical ICU Patients Alrefaey K Alrefaey, Ahmed S Marwa, Yassen M Amr, Zaghloul A Amgad
}

Department of Anesthesia and Surgical Intensive Care, Faculty of Medicine, Mansoura University, Egypt Correspondind author:Alrefaey K. Alrefaey, Email: refa3ey2@yahoo.com,Mobile: 00201064203475

\begin{abstract}
Introduction: Facing the recognized obstacles of chest computerized tomography (CT) and chest X-ray (CXR), lung ultrasound (LUS) is a promising alternative imaging tool gathering the benefits of real-time diagnosis with lower difficulties. This study aims to evaluate the effectiveness of LUS as a diagnostic modality in surgical critical care in contrast to chest $\mathrm{x}$-ray.

Patients and Methods: In this study, for each of the 92 included patients, when chest CT is indicated, CXR was requested and the BLUE protocol for LUS examination was initiated simultaneously. LUS data were recorded while analysis of CXR finding was performed by independent radiologist who was unaware of CT or LUS findings.

Results: For each patient, 4 lung areas were examined with LUS and compared to CXR. LUS showed higher specificity, sensitivity, positive predictive value (PPV), negative predictive value (NPV), and diagnostic accuracy (DA) of LUS in contrast to chest $x$-ray. A significantly lower time was required for LUS to reach the diagnosis compared to CXR (10.2 4 minutes vs. $33 \pm 17$ minutes respectively, $p$ less than 0.01$)$.

Conclusion: LUS is a useful tool in screening and diagnosis detection of various pulmonary problems in surgical ICU; it can be used as daily bedside diagnostic tool for the critically ill patients adding the use of other chest imaging modalities.
\end{abstract}

Key words: lung, ultrasound, complications, atelectasis.

\section{INTRODUCTION}

Postoperative pulmonary complications (POPC) are a major contributor to morbidity and mortality in the surgical patient with a high incidence reaching $40 \%$ of high-risk population ${ }^{(1,2)}$. Early detection of POPC is warranted to trigger early preventive and therapeutic interventions (3). Diagnosis of POPC is clinically suggested by findings like fever, cough and crepitation, and confirmed by thoracic imaging. For decades, thoracic imaging relied on routine bedside chest radiography (CXR), plus lung computed tomography (CT) when its indication is justified ${ }^{(3)}$.

Chest CT is generally accepted as the gold standard for the assessment of pulmonary structure and morphology. However, the risk of patient transfer and high radiation dosage restricts its use in critically ill patients to certain indications. On the other hand, CXR is a simple widely available modality, it carries a considerable technical defects that result in poor correlation between its findings and those of $\mathrm{CT}^{(4,5)}$.

Facing the previously mentioned barriers of both non contrast CT chest and CXR, LUS is proposed as a promising alternative imaging tool, gathering the benefits of real-time diagnosis and the availability as a bedside examination ${ }^{(6)}$.

The aim of this study is to evaluate the effectiveness of LUS as a diagnostic modality in surgical critical care sitting in contrast to chest $\mathrm{x}$-ray. We hypotheses that LUS is more sensitive and specific than chest $\mathrm{x}$-ray with less radiation exposure and more time-saving.

\section{PATIENTS AND METHODS}

The study included a cohort of 154 patients, admitted to surgical intensive care, Mansoura University Hospitals, and developed clinical data suggestive of POPC, including one or more of the following; fever, cough, sputum production, dyspnea, respiratory distress, and/or hypoxia. When chest CT is indicated for the patient, CXR and LUS assessment were simultaneously requested. Patients with chest wall deformities, thoracotomy patients and pregnant females were excluded from the study. 92 patients only could be examined by CT for difficulty to transfer or non-availability of CT.

\section{Ethical approval}

This study was conducted after approval of the Institutional Research Board, Mansoura University, Egypt (number: MS/15.12.42).

An informed consent was taken from the included patients or their guardians.

A portable ultrasound device (Siemens Acuson p300, Germany) was used for LUS examination (the BLUE protocol) ${ }^{(7,8)}$. While the patient was in supine position, the linear high-frequency transducer LA523 $(5-12 \mathrm{MHz})$ was used to examine lung sliding and subpleural consolidation. The convex transducer CA123 $(1-8 \mathrm{MHz})$ was applied for the rest of examination or from the start in case of obese patient. Scans were longitudinal with the probe orientation towards patient's head where the pleural line sought between two ribs (upper and lower).

Depth was adjusted according to patient size. Four clinical regions were assessed on each lung side (Figure 1). The Lung sliding sign, A-profile, Bprofile, pleural effusion and consolidations were 
screened. M-mode was used when there was a suspicion of pneumothorax to find stratosphere $\operatorname{sign}^{(9)}$. All data were documented as positive or negative and added to the patient specific report.

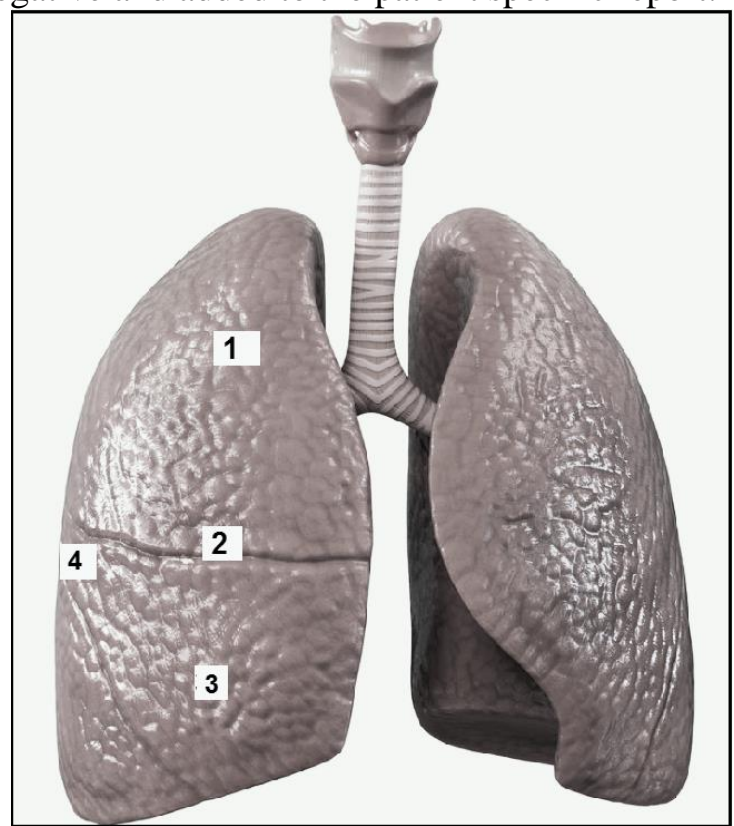

Figure 1: LUS examination Areas according to the study protocol

For all patients, AP-CXR was requested using a portable machine (Shimadzu Collimator R-20C portable x-ray device). Assessment of CXR was performed by independent radiologist who was unaware of CT or LUS findings. Time from requesting the CXR till the availability of the result for the ICU physician was documented. A Non-contrast Chest CT scanning was performed using Toshiba scanner Aquilion Prime TSX-303A (164-MCCT scanner) and was evaluated by an independent radiologist. For both CT and CXR report, the Nomenclature Committee of the Fleischner Society was used for defining CT findings ${ }^{(10)}$.

\section{Data collection and statistical analysis}

Patient name, age, sex, body weight and medical history were collected from patient medical file. The presence or absence of different lung pathologies by each diagnostic test (CXR or LUS) was documented including pneumonia, atelectasis, effusion, and pneumothorax. Time needed to perform the diagnostic test (CXR-LUS) was defined as the time elapsed from the order of the test till the interpretation of the results. Continuous variables were expressed as means \pm SD . Categorical data and nominal data were described as numbers (percentages). Both tests were compared to the gold standard test; Lung CT, to calculate sensitivity, specificity, PPV, NPV and DA. Time to reach definite diagnosis for each test was compared using independent sample t-test.

\section{RESULTS}

Descriptive data of the studied group is shown in table 1. For each patient, 4 lung areas were examined giving a total number of 368 comparisons between LUS and CXR (Figure 2).

Table 1: Patients characteristics of the included cohort. Data are presented as mean $\pm \mathrm{SD}$, absolute number and percentages

\begin{tabular}{lc}
\hline Age (years) & $40.6 \pm 14.7$ \\
Weight (kilograms) & $74.8 \pm 7.7$ \\
Height $(\mathrm{cm})$ & $164.5 \pm 5.2$ \\
BMI $\left(\mathrm{kg} / \mathrm{m}^{2}\right)$ & $27.7 \pm 3.5$ \\
Gender $(\mathrm{M} / \mathrm{F})$ & $72 / 20$ \\
Diagnosis: no (\%) & \\
Abdominal surgery: & $16(17 \%)$ \\
Neurosurgery: & $28(30 \%)$ \\
Polytrauma & $34(37 \%)$ \\
Others & $14(15 \%)$ \\
\hline
\end{tabular}

M: male, F: female. 


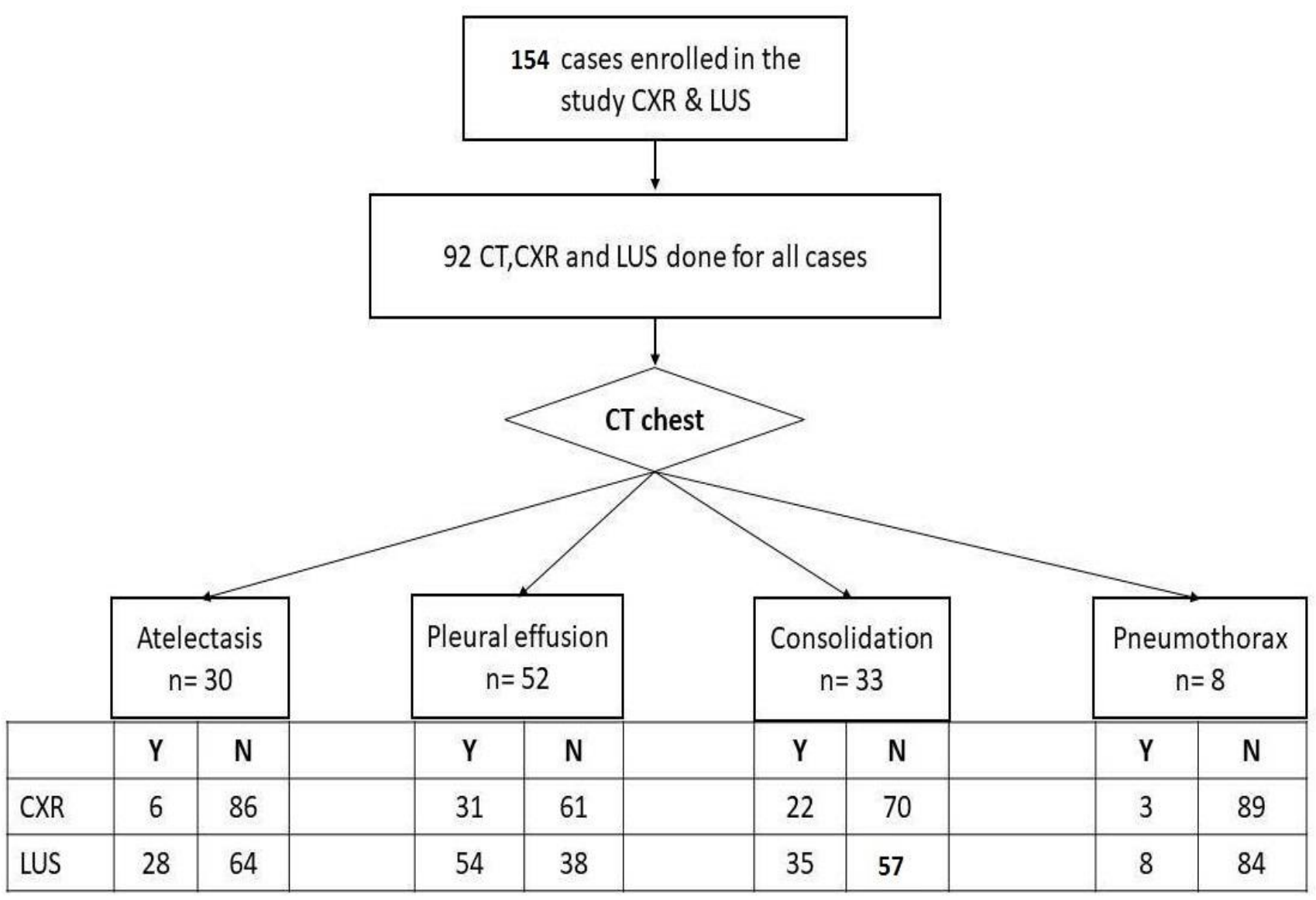

Figure 2: Chest CT, LUS and CXR findings in the study group

Results of the study show a higher specificity, sensitivity, positive predictive value (PPV), negative predictive value (NPV), and diagnostic accuracy (DA) of LUS in contrast to chest x-ray, see table 2 and figure 3.

Table 2: Diagnostic accuracy of LUS for POPC compared to CXR. Data are presented as percentage

\begin{tabular}{llllll}
\hline & Sensitivity & Specificity & PPV & NPV & DA \\
\hline Lung Ultrasound & & & & & \\
Pneumonia & $91 \%$ & $95 \%$ & $91 \%$ & $95 \%$ & $97 \%$ \\
Atelectasis & $90 \%$ & $100 \%$ & $100 \%$ & $95 \%$ & $97 \%$ \\
Pleural effusion & $100 \%$ & $100 \%$ & $100 \%$ & $100 \%$ & $100 \%$ \\
Pneumothorax & $100 \%$ & $100 \%$ & $100 \%$ & $100 \%$ & $97 \%$ \\
Chest X-Ray & & & & & \\
Pneumonia & $50 \%$ & $90 \%$ & $73 \%$ & $77 \%$ & $76 \%$ \\
Atelectasis & $23 \%$ & $100 \%$ & $100 \%$ & $72 \%$ & $74 \%$ \\
Pleural effusion & $66 \%$ & $100 \%$ & $100 \%$ & $72 \%$ & $73 \%$ \\
Pneumothorax & $100 \%$ & $100 \%$ & $100 \%$ & $100 \%$ & $100 \%$ \\
\hline LUS: & & & & & \\
\hline
\end{tabular}

LUS: lung ultrasound, POPC: postoperative pulmonary complications, CXR: chest X ray, PPV: positive predictive value, NPV: negative predictive value, DA: Diagnostic accuracy. 


\section{Cases recruited for study $\mathrm{n}=\mathbf{1 5 4}$}

\section{Cases completed the study protocol $(n=92)$ Chest CT, LUS and CXR were done}

\begin{tabular}{|c|c|c|c|c|c|c|c|c|}
\hline $\begin{array}{c}\text { Diagnosed } \\
\text { by CT }\end{array}$ & \multicolumn{2}{|c|}{$\begin{array}{c}\text { Atelectasis } \\
(\mathbf{n}=\mathbf{3 1})\end{array}$} & \multicolumn{2}{c|}{$\begin{array}{c}\text { Effusion } \\
(\mathbf{n}=\mathbf{5 4 )}\end{array}$} & \multicolumn{2}{c|}{$\begin{array}{c}\text { Pneumonia } \\
(\mathbf{n}=\mathbf{3 2})\end{array}$} & \multicolumn{2}{c|}{$\begin{array}{c}\text { Pneumothorax } \\
(\mathbf{n}=\mathbf{8})\end{array}$} \\
\hline & CXR & LUS & CXR & LUS & CXR & LUS & CXR & LUS \\
\hline TT & 7 & 28 & 29 & 54 & 16 & 32 & 2 & 8 \\
\hline FT & 0 & 0 & 0 & 0 & 6 & 3 & 0 & 0 \\
\hline TF & 61 & 61 & 38 & 38 & 54 & 57 & 80 & 81 \\
\hline FF & 24 & 3 & 15 & 0 & 16 & 3 & 9 & 0 \\
\hline
\end{tabular}

Figure 3: Comparison between CXR and US finding in relation to chest CT findings. TT: True-true, FT: Falsetrue, TF: true-false, FF: False -false.

Time needed to reach diagnosis after CXR and after LUS was documented and compared (Figure 4). A significantly lower time was required for LUS to reach diagnosis compared to CXR (10.2 4 minutes vs. $33 \pm 17$ minutes respectively, $p$ less than 0.01 ).

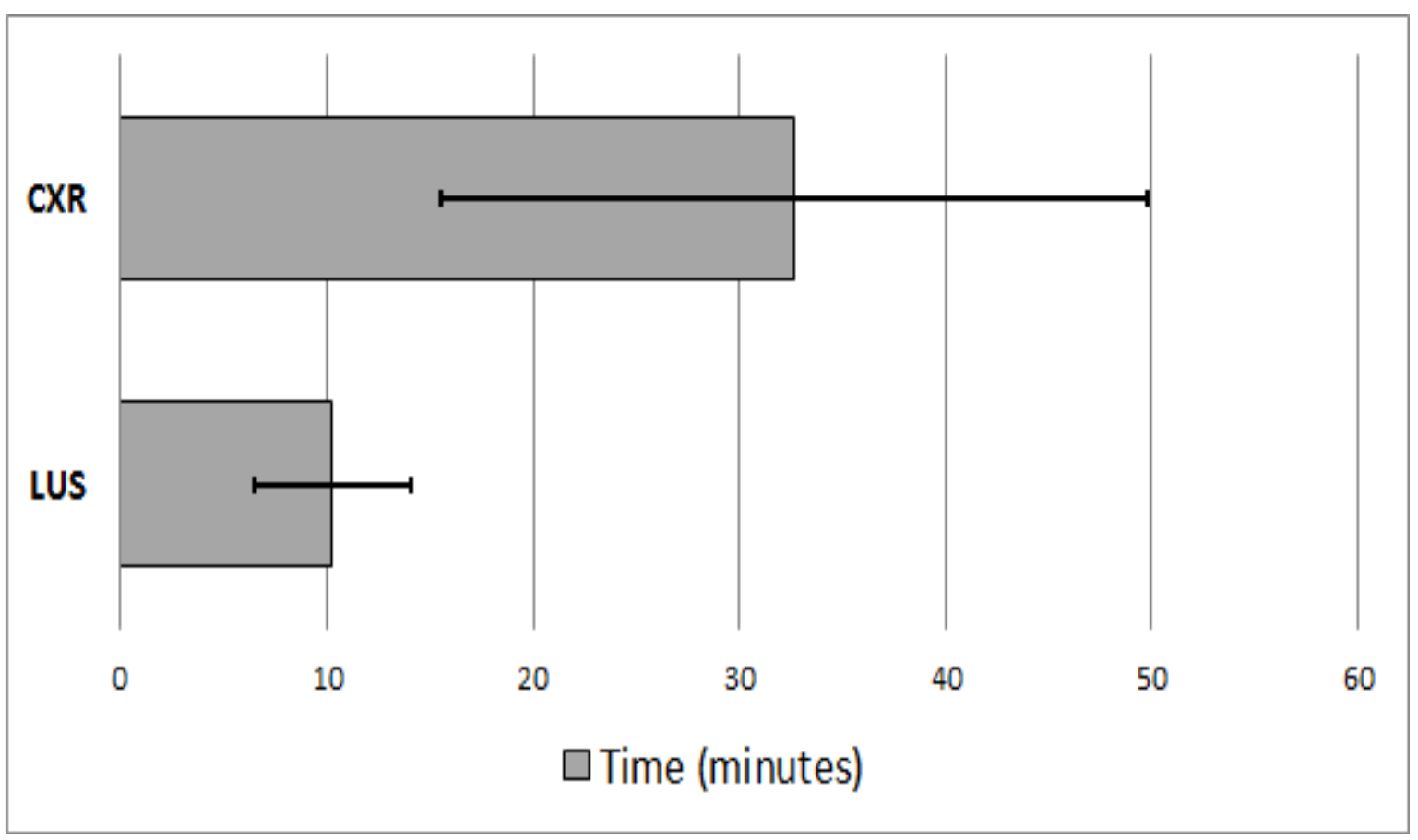

Figure 4: Time needed to reach diagnosis after CXR and after LUS. 


\section{DISCUSSION}

The present study aimed to evaluate the potential role of LUS as screening tool for the diagnosis of POPC, as pleural effusions, pneumonia, atelectasis, pulmonary edema and pneumothorax, in the surgical ICU patients. The study recruited 154 critically ill patients, 62 could not complete the study protocol due to non-ability to perform CT chest. The remaining 92 patients were triply imaged using CT, CXR and LUS. The results of our study revealed a significantly greater agreement between CT and LUS compared to CXR interpretation results.

POPC is generally considered as the second most common cause of perioperative morbidity after cardiovascular events. Its occurrence varies from $2 \%$ after minor procedures to $40 \%$ in high risk patients ${ }^{(1}$, 11). Early diagnosis can help to start early preventive and therapeutic measures to and to improve patient outcome $^{(1,2)}$.

Despite lung CT is the gold standard for chest radiology, decision to perform the test must be balanced against the risk of patient transfer and the high radiation exposure. CT is mostly considered when inherent limitations of CXR result in unclear diagnosis $(4,12)$. Consequently, chest $\mathrm{X}$ ray was used for screening, diagnosis and follow up of pulmonary complications in critically ill patients especially if attached to ventilator ${ }^{(5)}$. Currently, LUS is progressively utilized in clinical practice ${ }^{(13-15)}$, as it allows bedside visualization of the lungs. LUS could be a practical, bedside diagnostic tool in critically ill patients because of relatively high sensitivity, along with accessibility and simplicity of the technique. This allows prompt and fast response to enable a critical care physicians to manage a majority of critical situations ${ }^{(14,16)}$.

Several studies investigated the application of LUS. Xirouchaki and his colleagues compared the diagnostic performance of both LUS on 42 mechanically ventilated patients. LUS was significantly more sensitive and specific than bedside CXR in recognizing various lung pathologies ${ }^{(4)}$. Another study conducted upon 130 patients comparing LUS results to those of CT chest and plain CXR. The results confirmed the advantage of LUS over CXR as a diagnostic modality ${ }^{(17)}$.

Atelectasis is one of the most encountered abnormalities in chest radiology after major abdominal surgery with an incidence that can reach $90 \%$ even in previously normal patients ${ }^{(2,11)}$. Early interventions to expand the affected lung areas are extremely valuable to prevent the development of postoperative pneumonia $^{(1)}$. Daniel A. Lichtenstein and his colleagues showed that with absent lung sliding, LUS can effectively diagnose complete atelectasis before the occurrence of any radiological changes ${ }^{(18)}$.

In our study, visualization lung consolidation, hepatization, or alveolar interstitial syndromes with positive clinical findings was used to diagnose pneumonia. Corresponding to many studies ${ }^{(19-21)}$, our results confirmed that the use of LUS over CXR in diagnosis of pneumonia demonstrated higher specificity, sensitivity and DA. For cases admitted to ER with suspected CAP, LUS dependent diagnosis agreed with CT finding in nearly all the diagnosed cases especially at early pneumonia development ${ }^{(22,23)}$, an advantage that can elicit earlier therapeutic interventions. In comparison to other diagnostic tests, LUS can differentiate causes of lung consolidation including atelectasis, acute respiratory distress syndrome (ARDS) and lung contusions ${ }^{(24)}$.

Along with our results, LUS was frequently used for the diagnosis of pleural effusion. In a prospective study of critically ill patients with acute respiratory distress syndrome, the DA of LUS for pleural effusions was superior (93\%) contrasted to auscultation (61\%) and AP CXR (47\%), using chest CT as the reference standard. 4 studies calculated a pooled sensitivity and specificity of ultrasound for detection of pleural effusions as 93\% (95\% CI: 89\%-96\%) and 96\% (95\% CI: 95\%-98\%), respectively ${ }^{(25)}$.

Time required to reach diagnosis using point-ofcare LUS (POC-LUS) is significantly lower than that needed for CXR. During this time, earlier treatment protocols can be imitated resulting in an improved patient outcome. Average time required for an X-ray film to be interpreted may reach 2 hours in some studies compared to few minutes when US machine is readily available and the practice is well settled in the place ${ }^{(26)}$

In addition to the above clinical conditions, LUS was employed and compared to CXR for the assessment of pneumothorax, pulmonary edema, and $\operatorname{ARDS}^{(12,15,27-29)}$. Based on the high efficacy of LUS in the diagnosis of pneumothorax, the Eastern Association for the Surgery of Trauma gave a level 2 recommendation for the use of ultrasound to identify traumatic pneumothorax in its practice management guidelines ${ }^{(30)}$. Likewise, bedside LUS was perceived as a conceivably helpful way to evaluate pulmonary congestion. A meta-analysis incorporating 1075 patients from seven different studies revealed that Blines detected by LUS are useful for the identification of cardiogenic origin of pulmonary edema ${ }^{(31)}$. Economic advantage of LUS versus CXR in ICU setting indicated by the lower cost for chest imaging especially when the patient condition is dynamic and repeated follow up is indicated ${ }^{(32,33)}$.

The study has some limitations. Firstly, the time lag between the performance of LUS, CXR and CT with lag for more than 10 minutes in many cases, which when a pneumothorax is taken as example, enough time to enlarge, however, management of such lifethreatening situation depends on clinical diagnosis without waiting for the result of any of the 3 studied tests. Ultrasound probe applied to the posterior zone of the chest of a bedridden, immobilized, intubated, or unconscious patient was an obstacle as many times it couldn't be brought between the patient and the bed, a 
problem that exists also during positioning of the $\mathrm{X}$ ray film and is over weighted by a higher risk of patient transfer if compared to CT examination.

In conclusion, LUS is a useful tool in screening and diagnosis detection of various pulmonary problems such as pleural effusion, atelectasis, pneumonia pneumothorax, and pulmonary edema. It can be used as daily bedside diagnostic tool for the critically ill patients adding the use of other chest imaging modalities.

\section{REFERENCES}

1. Fernandez-Bustamante A, Frendl G, Sprung J et al. (2017): Postoperative pulmonary complications, early Mortality, and hospital stay following non-cardiothoracic Surgery. JAMA Surg., 152-7

2. Miskovic A, Lumb A (2017): Postoperative pulmonary complications. Br J Anaesth. , (118): 317-34

3. Touw HR, Parlevliet KL, Beerepoot M et al. (2018): Lung ultrasound compared with chest $\mathrm{X}$-ray in diagnosing postoperative pulmonary complications following cardiothoracic surgery: a prospective observational study. Anaesthesia, (73): 946-54

4. Xirouchaki N, Magkanas E, Vaporidi K (2011): Lung ultrasound in critically ill patients: Comparison with bedside chest radiography. Intensive Care Med., (37): 1488-93

5. Rubinowitz AN, Siegel MD, Tocino I (2007): Thoracic Imaging in the ICU. Crit Care Clin., (23): 539-73

6. Gardelli G, Feletti F, Nanni A et al. (2012): Chest ultrasonography in the ICU. Respir Care, (57): 773-81

7. Lichtenstein D, Mezière G (2008): Relevance of lung ultrasound in the diagnosis of acute respiratory failure the BLUE protocol. Chest, (134): 117-25

8. Lichtenstein D (2015): BLUE-Protocol and FALLSProtocol: Two applications of lung ultrasound in the critically ill. Chest, (147): 1659-70.

9. Miller A (2016): Practical approach to lung ultrasound. BJA Education, 16(2): 39-45

10. Hansell D, Bankier A, MacMahon H et al. (2008): Fleischner Society: Glossary of terms for thoracic imaging. https://pubmed.ncbi.nlm.nih.gov/18195376/

11. Kelkar $K$ (2015): Post-operative pulmonary complications after non-cardiothoracic surgery. Indian J Anaesth., (59): 599-605

12. Khalil M, ELMaraghy A, Yousef Y (2015): Could chest ultrasonography replace routine chest X-rays in mechanically ventilated patients? Egypt J Chest Dis Tuberc., (64): 857-63

13. Bostantzoglou C, Moschos C (2013): Introduction to transthoracic ultrasound for the pulmonologist. Pneumon., (26): 229-34

14. Alzahrani S, Al-Salamah M, Al-Madani $\mathrm{W}$ et al. (2017): Systematic review and meta-analysis for the use of ultrasound versus radiology in diagnosing of pneumonia.

https://www.ncbi.nlm.nih.gov/pmc/articles/PMC532890 6/

15. Elmahalawy I, Doha N, Ebeid O et al. (2017): Role of thoracic ultrasound in diagnosis of pulmonary and pleural diseases in critically ill patients. Egypt J Chest Dis Tuberc., (66): 261-6
16. Exhibit E, Guarnieri T, Ramanzin A et al. (2017): Thoracic Imaging: correlation of sonographic signs at Lung Ultrasound with Chest Radiography and Computed Tomography Images, 1-26

17. Koenig S, Mayo P, Volpicelli G et al. (2020): Lung Ultrasound Scanning for Respiratory Failure in Acutely Ill Patients. Chest, 158(6): 2511-6

18. Lichtenstein DA, Lascols N, Prin S et al. (2003): The "lung pulse": An early ultrasound sign of complete atelectasis. Intensive Care Med., (29): 2187-92

19. Ye X, Xiao H, Chen B, Zhang S (2015): Accuracy of lung ultrasonography versus chest radiography for the diagnosis of adult community-acquired pneumonia: Review of the literature and meta-analysis. PLoS One, (10): $1-9$

20. Amatya Y, Rupp J, Russell F et al. (2018): Diagnostic use of lung ultrasound compared to chest radiograph for suspected pneumonia in a resource-limited setting. Int $\mathbf{J}$ Emerg Med., (11): 8

21. Alkhayat K, Alam-Eldeen M (2014): Value of chest ultrasound in diagnosis of community acquired pneumonia. Egypt J Chest Dis Tuberc., (63): 1047-51

22.Parlamento S, Copetti R, Bartolomeo S. (2009): Evaluation of lung ultrasound for the diagnosis of pneumonia in the ED. Am J Emerg Med., (27): 379-84

23. Bourcier J, Paquet J, Seinger $M$ et al. (2014): Performance comparison of lung ultrasound and chest $\mathrm{X}$ ray for the diagnosis of pneumonia in the ED. Am J Emerg Med., (32): 115-8

24. Blaivas M (2012): Lung ultrasound in evaluation of pneumonia. J Ultrasound Med., (31): 823-6

25. Soni N, Franco R, Velez M et al. (2015): Ultrasound in the diagnosis and management of pleural effusions. J Hosp Med., (10): 811-6

26. Amatya Y, Rupp J, Russell F et al. (2018): Diagnostic use of lung ultrasound compared to chest radiograph for suspected pneumonia in a resource-limited setting. Int $\mathbf{J}$ Emerg Med., 11-8

27. Cattarossi L, Copetti R, Brusa G et al. (2016): Lung Ultrasound Diagnostic Accuracy in Neonatal Pneumothorax. Can Respir J., 6515069.

28. Lee F (2016): Lung ultrasound-a primary survey of the acutely dyspneic patient. J Intensive Care, (4): 57

29. Dubinsky T, Shah H, Sonneborn $R$ et al. (2017): Correlation of B-Lines on Ultrasonography with interstitial lung disease on chest radiography and CT imaging. Chest, (152): 990-8

30. Mowery N, Gunter O, Collier B et al. (2011): Practice management guidelines for management of hemothorax and occult pneumothorax. J Trauma, 70(2): 510-8

31. Deeb M, Barbic S, Featherstone $R$ et al. (2014) : Point-of-care ultrasonography for the diagnosis of acute cardiogenic pulmonary edema in patients presenting with acute https://pubmed.ncbi.nlm.nih.gov/25176151/

dyspnea.

32. Deeb M, Barbic S, Featherstone R et al. (2014): Pointof-care Ultrasonography for the Diagnosis of Acute Cardiogenic Pulmonary Edema in Patients Presenting with Acute Dyspnea: A Systematic Review and Metaanalysis. Acad Emerg Med., (21): 843-52

33. Brogi E, Bignami E, Sidoti A et al. (2017): Could the use of bedside lung ultrasound reduce the number of chest $\mathrm{x}$-rays in the intensive care unit? Cardiovasc Ultrasound, 15-23. 